\title{
On modeling the large strain fracture behaviour of soft viscous foods ${ }^{\circ}$
}

Cite as: Phys. Fluids 29, 121610 (2017); https://doi.org/10.1063/1.4993754

Submitted: 30 June 2017 . Accepted: 19 October 2017 . Published Online: 29 November 2017

\section{G. Skamniotis, M. Elliott, and M. N. Charalambides}

\section{COLLECTIONS}

F This paper was selected as Featured
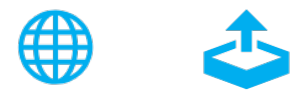

\section{ARTICLES YOU MAY BE INTERESTED IN}

Frictional shear thickening in suspensions: The effect of rigid asperities

Physics of Fluids 29, 121607 (2017); https://doi.org/10.1063/1.4989929

Hydrodynamics experiments with soap films and soap bubbles: A short review of recent experiments

Physics of Fluids 29, 111113 (2017); https://doi.org/10.1063/1.4986003

Preface to Special Topic: Papers from the Institute of Non-Newtonian Fluid Mechanics Meeting, Lake Vyrnwy, 2017

Physics of Fluids 29, 121401 (2017); https://doi.org/10.1063/1.5018585

\section{AIP Advances Fluids and Plasmas Collection}




\title{
On modeling the large strain fracture behaviour of soft viscous foods
}

\author{
C. G. Skamniotis, ${ }^{1, a)}$ M. Elliott, ${ }^{2}$ and M. N. Charalambides ${ }^{1, a)}$ \\ ${ }^{1}$ Department of Mechanical Engineering, Imperial College London, London SW7 2AZ, United Kingdom \\ ${ }^{2}$ Mars Petcare, Oakwell Way, Birstall, Batley WF17 9LU, United Kingdom
}

(Received 30 June 2017; accepted 19 October 2017; published online 29 November 2017)

\begin{abstract}
Mastication is responsible for food breakdown with the aid of saliva in order to form a cohesive viscous mass, known as the bolus. This influences the rate at which the ingested food nutrients are later absorbed into the body, which needs to be controlled to aid in epidemic health problems such as obesity, diabetes, and dyspepsia. The aim of our work is to understand and improve food oral breakdown efficiency in both human and pet foods through developing multi-scale models of oral and gastric processing. The latter has been a challenging task and the available technology may be still immature, as foods usually exhibit a complex viscous, compliant, and tough mechanical behaviour. These are all addressed here through establishing a novel material model calibrated through experiments on starch-based food. It includes a new criterion for the onset of material stiffness degradation, a law for the evolution of degradation governed by the true material's fracture toughness, and a constitutive stress-strain response, all three being a function of the stress state, i.e., compression, shear, and tension. The material model is used in a finite element analysis which reproduces accurately the food separation patterns under a large strain indentation test, which resembles the boundary conditions applied in chewing. The results lend weight to the new methodology as a powerful tool in understanding how different food structures breakdown and in optimising these structures via parametric analyses to satisfy specific chewing and digestion attributes. Published by AIP Publishing. https://doi.org/10.1063/1.4993754
\end{abstract}

\section{INTRODUCTION}

Recently, there has been a multidisciplinary interest in feeding mechanics (Wang and Chen, 2017) and particularly chewing efficiency, since the latter relates to the ability of organisms to breakdown and digest food (Eng et al., 2013; Bierer, 2007; Drechsler and Ferrua, 2016; and Berthaume, 2016b; 2016a). In humans, advances in thermo-mechanical food processing alter food mechanical properties towards less masticatory effort (Eng et al., 2013; Le Révérend and Hartmann, 2014; and Le Révérend et al., 2016). In contrast, animal species and especially domesticated pets show a wide range of masticatory characteristics due to a marked variation in skull morphology (Berthaume, 2016b; Bierer, 2007; and Skamniotis et al., 2016), imposing dietary specializations (Luke and Lucas, 1983; Kupczik and Stynder, 2012; and Berthaume et al., 2013). This complicates the formulation of palatable food products according to the respective needs of different pet breeds and for this reason the degree of pet food optimisation may still be limited (Skamniotis et al., 2016; 2017a).

Breakdown efficiency is of major importance for the overall digestion process (Bornhorst and Singh, 2012; Kong and Singh, 2008; and Drechsler and Ferrua, 2016). Inadequate oral breakdown can lead to maldigestion (Pera et al., 2002). Chewing increases the total surface area of the food item, in order to facilitate further breakdown in the stomach through peristaltic waves (Ramanamurthy et al., 2013) and enzymatic

\footnotetext{
a)Electronic addresses: c.skamniotis13@imperial.ac.uk and m.charalambides @ imperial.ac.uk
}

disintegration (Berthaume, 2016a; Bornhorst and Singh, 2012; and Cleary et al., 2015), followed by nutrient absorption in the small intestine (Wang et al., 2010 and Lentle et al., 2013). While so far the gastrointestinal system has been approached through fluid dynamics (Cleary et al., 2015; Kozu et al., 2010; and Wang et al., 2010), the overall process is heavily affected by the initial number of food fragments and the associated fragment size distribution in the bolus, which is of course a function of food texture (Bornhorst and Singh, 2012; Wang and Chen, 2017; Drechsler and Ferrua, 2016; and Loret et al., 2011). Hiiemae et al. (1996) showed that increasing food hardness implies smaller fragment sizes and a larger number of bites/chews. Moreover, large and hard solid particles demonstrate low gastric emptying rates, as opposed to small and soft in which the rates are profoundly higher (Kong and Singh, 2008 and Drechsler and Ferrua, 2016); the same trend corresponds between high and low viscosity fluids (Moxon et al., 2016 and Kozu et al., 2010). Controlling the gastric emptying rate receives considerable attention because it associates with metabolic effects and the management of obesity (Cohen et al., 2003 and Cardoso-Júnior et al., 2007), both in humans and pets (German et al., 2010). It is also strongly related to diabetes and stomach disorders such as dyspepsia (Rayner et al., 2001). Summarising, there is an increasing need in models of food breakdown not only to address a healthy oral processing but also to aid in a controlled gastrointestinal digestion (Moxon et al., 2016; Taghipoor et al., 2012; and Norton et al., 2007).

Historically, probabilistic food particle size distribution functions were proposed (Epstein, 1947; Lucas and Luke, 1983a; 1983b; and Voon et al., 1986) and some of them 
correlated well with in vivo chewing data (Baragar et al., 1996). However, none of them took into account any of the food physical properties. These were considered later, through establishing fundamental relations between food fracture resistance and mechanical properties such as the elastic modulus and toughness (Vincent, 2004; Agrawal and Lucas, 2003; and Agrawal et al., 1997). Yet, these were limited to a linear elastic hard food response upon a simple bite geometry (Agrawal and Lucas, 2003 and Agrawal et al., 1997). Recent developments include complex hyperviscoelastic constitutive laws for foods (hyperelastic rubber models combined with viscoelastic models such as the Prony series), which are used to relate mechanical properties to sensory attributes and breakdown behaviour (Mohammed et al., 2013; Charalambides et al., 2002; Goh et al., 2005; and Gamonpilas et al., 2009). These material constitutive laws were fed into finite element (FE) models of soft food separation, in the form of wire cutting and blade indentation (McCarthy et al., 2010; Gamonpilas et al., 2009; and Goh et al., 2005). However, these techniques postulate a predefined fracture path (Vandenberghe et al., 2017), which is certainly not the case for oral (Skamniotis et al., 2016) and gastrointestinal breakdown (Cleary et al., 2015). Furthermore, food separation was only considered in simple tension, while the understanding of food breakdown under multiaxial states, i.e., compression, shear, and tension, remains unclear.

Undoubtedly, modeling arbitrary fracture patterns for non-linear, rate dependent foods remains a challenging task (Skamniotis et al., 2016 and Wang and Chen, 2017). Particularly soft foods undergo large deformations when masticated, which causes significant topological changes and non-linearity in numerical analyses (Simulia, 2013 and Skamniotis et al., 2017a). Harrison et al. predicted promising results of food breakdown in human mastication, using the Smoothed Particle Hydrodynamics (SPH) method (Harrison et al., 2014a). However, a simple elastic followed by perfectly plastic stressstrain behaviour and a simplistic fracture criterion were only defined for the food, while the toughness and teeth-food interfacial friction parameters were not considered. Although the SPH method handles easily large deformations and complex motions due to its meshless formulation (Harrison et al., 2014a), the computational efficiency and accuracy are often compromised (Mohagheghian et al., 2017; Simulia, 2013; Liu et al., 2007; and Skamniotis et al., 2017a).
Recently, the authors introduced a more advanced FE model of breakdown in starch-based pet foods, with greater potential for application in parametric studies of texture and product optimisation (Skamniotis et al., 2016). This is because the model enabled the use of multiple frictional conditions and more importantly the inclusion of rate dependency and fracture toughness in the food material model definition. However, a single stress state was considered as the material model was calibrated only based on tensile data. In this work, we extend our previous material model reported in Skamniotis et al. (2016) to account for the dependence of both the stressstrain response and fracture toughness on the stress state. This involves new compression, shear, and tensile data, as well as a new and more accurate toughness value; the latter was derived in our very recent work reported in Skamniotis et al. (2017b) via the essential work of fracture method (Bárány et al., 2010). Finally, the novel material model is validated through an independent cylindrical indentation experiment which resembles the boundary conditions applied in chewing.

\section{MATERIALS AND METHODS}

\section{A. Material}

The food was provided in the form of sheet and square cross section extrudates by Mars Petcare UK, as depicted in Figs. 1(a) and 1(b), respectively. The material consisted of gelatinized cereal starch (15-20 $\mu \mathrm{m}$ raw granule size) at $45 \% \mathrm{w} / \mathrm{w}$, crude fibre components $(200-500 \mu \mathrm{m}$ length and 20-30 $\mu \mathrm{m}$ diameter) at $2.5 \% \mathrm{w} / \mathrm{w}$, and dissolved protein (20-23 kDa molecular weight) at $4 \% \mathrm{w} / \mathrm{w}$, while the rest was water. Previous test results reported in Skamniotis et al. (2016) indicated a homogeneous, isotropic behaviour. Nozzle size effects on texture between the two extrudate profiles (see Fig. 1) were minimised via using a common cross sectional area, $A_{o}=143 \pm 2.6 \mathrm{~mm}^{2}$ (Amuthakkannan et al., 2013).

Material stiffening and embrittlement effects were found to occur in two distinct ways. In the short term, upon exposure to ambient air, the moisture loss led to a significantly harder texture, within one day (Moscicki et al., 2013). To alleviate this effect, the samples were strictly kept sealed after production until testing. At the longer time scale, i.e., three weeks storage time, the starch re-crystallization mechanism, known as ageing, caused a noticeable increase in the material stiffness

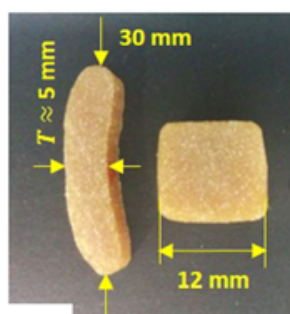

(a)

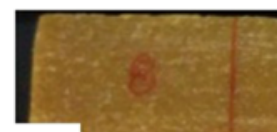

(c)

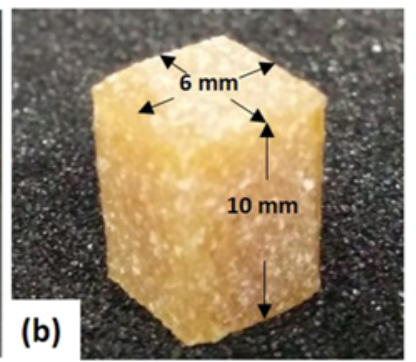

(b)
FIG. 1. (a) Cross sectional view of provided extrudate profiles: sheet (left) and square (right), (b) rectangular specimen for uniaxial compression cut from square profile extrudates; original dimensions denote original specimen height, $H_{o}=10 \mathrm{~mm}$, and original square edge length, $D_{o}=6 \mathrm{~mm}$. (c) Dumbbell specimen for uniaxial tension cut from sheet profile extrudates; dimensions are denoted. 
(Moscicki et al., 2013). For consistency, all the test data used to calibrate the material model (presented later in Sec. III) were obtained at approximately three weeks following production and on three consecutive days of testing; these results are referenced as "aged" texture data, throughout. For comparison, additional data were obtained at three days after production, referenced as "fresh" texture results, while other test data obtained at three months after production are referenced as "over-aged" data. All the samples were stored and tested at a constant temperature of $20^{\circ} \mathrm{C}$ and relative humidity of $50 \%$.

\section{B. Experimental procedure}

All the tests were performed on a single column Zwick Roell universal testing machine with a $1 \mathrm{kN}$ load cell capacity. Compression and tensile monotonic stress-strain data were obtained both for the aged and fresh textures. The compression tests were performed on cuboid specimens, as shown in Fig. 1(b), cut via square profile dies and a mechanical press on the square profile extrudates. The strain rate dependency was assessed via monotonic tests up to large true strain magnitudes of 2, at the five constant true strain rates: $0.0001 \mathrm{~s}^{-1}$, $0.01 \mathrm{~s}^{-1}, 0.1 \mathrm{~s}^{-1}, 1 \mathrm{~s}^{-1}$, and $5 \mathrm{~s}^{-1}$. Variation often found in foodstuffs within single batches (Goh, 2002) was addressed via performing five valid test replicates; this number was found to provide a statistically valid sample both for compression and tension (Charalambides et al., 2006). All the data will be plotted throughout as mean values, using standard deviation as error bars. Further investigation involved relaxation tests for the aged texture at the strain magnitudes of $0.1,0.2$, and 0.5 , which were held constant for $20 \mathrm{~min}$, while the stress decay was monitored; the initial loading was performed via a ramp strain at $0.01 \mathrm{~s}^{-1}$ rate while two test repeats were performed. The true (Cauchy) stress, $\sigma$, versus true (Hencky) strain, $\varepsilon$, data were calculated via

$$
\left.\begin{array}{lc}
\text { (a) } & \sigma=F / A_{i} \\
\text { (b) } & \varepsilon=\ln \left(H_{i} / H_{o}\right)
\end{array}\right\}
$$

where $A_{i}$ is the instantaneous cross sectional area of the specimen, $F$ is the corresponding force applied, and $H_{o}$ is an original reference dimension of the specimen (height in compression and gauge length in tension) together with its deformed value, $H_{i}$. Equation (1) is based on a fully incompressible behaviour (Poisson's ratio of $v=0.5$ ) previously found for this material (Skamniotis et al., 2016). Note that although it gives $\varepsilon<0$ for compression, for simplicity, the term "strain" and the $\varepsilon$ values used in the stress-strain plots refer to strain magnitude. Sample-platen interfacial friction effects on the results were minimised via attaching polytetrafluoroethylene (PTFE) sheets ( $0.5 \mathrm{~mm}$ thickness) on the platens (Forte et al., 2016 and Charalambides et al., 2006). The PTFE surfaces were also smeared with silicon oil of $100000 \mathrm{cST}\left(=0.1 \mathrm{~m}^{2} / \mathrm{s}\right)$ viscosity (Goh et al., 2005), high enough to prevent the lubricant from being squeezed out during compression as well as to reduce its absorption into the sample. While the "squeeze out" effects were inevitable at large strains, preliminary tests verified that the specific lubricant did not have any adverse effect on the measured properties, i.e., the absorption into the sample was insignificant. Specimen geometry criteria against friction effects proposed in Charalambides et al. (2005) and Goh and Sherman (1987) were also considered via using a sufficiently high specimen height over square edge ratio, $H_{o} / D_{o}$ $>1.6$, i.e., $H_{o}=10 \mathrm{~mm}$ and $D_{o}=6 \mathrm{~mm}$, as indicated in Fig. 1(b).

The tensile tests were conducted on dumbbell specimens, as shown in Fig. 1(c), obtained via cutting sheet profile extrudates through standard specimen dies and a mechanical press. The specimen thickness, width, and grip-to-grip length were approximately $5 \mathrm{~mm}, 12 \mathrm{~mm}$, and $100 \mathrm{~mm}$, respectively, while a gauge length of $40 \mathrm{~mm}$ was marked and subsequently optically tracked during the tests, as described in Skamniotis et al. (2016) and Skamniotis et al. (2017b). The rate dependent data were obtained through monotonic tests up to ultimate specimen separation at the rates of $0.0001 \mathrm{~s}^{-1}, 0.001 \mathrm{~s}^{-1}, 0.01 \mathrm{~s}^{-1}$, $0.1 \mathrm{~s}^{-1}$, and $1 \mathrm{~s}^{-1}$ and five valid test repeats per rate. Stress relaxation data were obtained for the aged texture, through monitoring the stress decay for 20 minutes, at the three constant true strains of $0.03,0.06$, and 0.08 ; the initial loading was performed through a ramp strain at $0.01 \mathrm{~s}^{-1}$ rate while two test repeats were performed. At higher strains, i.e., $0.11,0.13$, and 0.15 , tensile failure occurred after a certain hold time and the relaxation plots are not presented. However, the data were considered for the tensile strain at break criterion presented later in Sec. III B. This criterion was further verified via three creep failure data points obtained through applying a ramp stress at $0.5 \mathrm{MPa} / \mathrm{s}$ rate, at the levels of $1.4 \mathrm{MPa}, 1.8 \mathrm{MPa}$, and $2.3 \mathrm{MPa}$.

\section{Numerical study}

The experimental data derived through the above procedures were utilized in calibrating the material constitutive law (presented in Sec. IV) used in the food breakdown FE simulations (presented in Sec. V). The numerical study assessed the capability of this constitutive law in predicting breakdown, through reproducing the experimental large strain indentation response of the food. This included in situ SEM cylindrical indentation tests, allowing for SEM observations of the evolution of damage on the sample's surface, while applying multi-axial states, particularly compression and shear (Gamonpilas et al., 2010), as experienced during oral breakdown (Skamniotis et al., 2017a).

Although the constitutive law is fitted to the stress-strain response both for the fresh and aged textures, it was not judged necessary to demonstrate breakdown simulation results for both textures. Furthermore, introducing damage in the indentation FE simulations required an additional critical toughness value, which was derived experimentally in our very recent work (Skamniotis et al., 2017b) only for the aged texture; thus breakdown is predicted only for the aged samples. However, indentation tests were still conducted for a wide range of textures, i.e., fresh, aged, and over-aged, in order to quantify the effect of ageing on the overall breakdown response.

The numerical study was performed via the commercial FE software ABAQUS 2016 (Simulia, 2013) and by using eight processor cores (Intel i7 CPU at 3.2 GHz). Details on the experimental apparatus and numerical model are provided in Sec. V A. 


\section{EXPERIMENTAL RESULTS}

\section{A. Stress-strain data}

Figures 2(a) and 2(b) depict the average monotonic stressstrain, $\sigma-\varepsilon$, curves for the various rates in compression and tension, respectively, for the fresh texture, together with the viscoplastic model fit; the latter is discussed in Sec. III A. In the same order, Figs. 2(c) and 2(d) illustrate the data for the aged texture along with the viscoplastic model fit. Note that for these uniaxial states, the $\sigma-\varepsilon$ data are equal to the equivalent stress versus equivalent strain, $\sigma_{e q}-\varepsilon_{e q}$, data, which are considered in the viscoplastic model presented later in Sec. III A. Also, the data points for the $0 \mathrm{~s}^{-1}$ rate in Figs. 2(c) and 2(d) correspond to the long term stresses obtained from relaxation tests shown in Figs. 2(e) and 2(f), at the corresponding constant strains.
The average typical variabilities for each rate were $6 \%$ in Fig. 2(a) and $8 \%$ in Fig. 2(b), between the five repeats. The respective results for the aged texture are shown in Figs. 2(c) and 2(d). The corresponding variabilities were $9 \%$ in Fig. 2(c) and $10 \%$ in Fig. 2(d). In agreement with a previous study (Skamniotis et al., 2016), a non-linear strong rate dependent behaviour is observed in both textures, as well as a time dependent tensile strain at break, $\varepsilon_{f}$ [denoted as " $x$ " in Figs. 2(b) and 2(d)]. Micro-cracks perpendicular to the tensile strain were also apparent, both in compression and tension; note that in compression the tensile strain occurs laterally due to material expansion. Overall, for the same strain magnitude the tensile stresses are higher than the compressive, which may be attributed to a weaker fibre stiffening effect in compression. Clearly, the fresh material displays a softer response owing to lower stresses in both tension and compression. On the
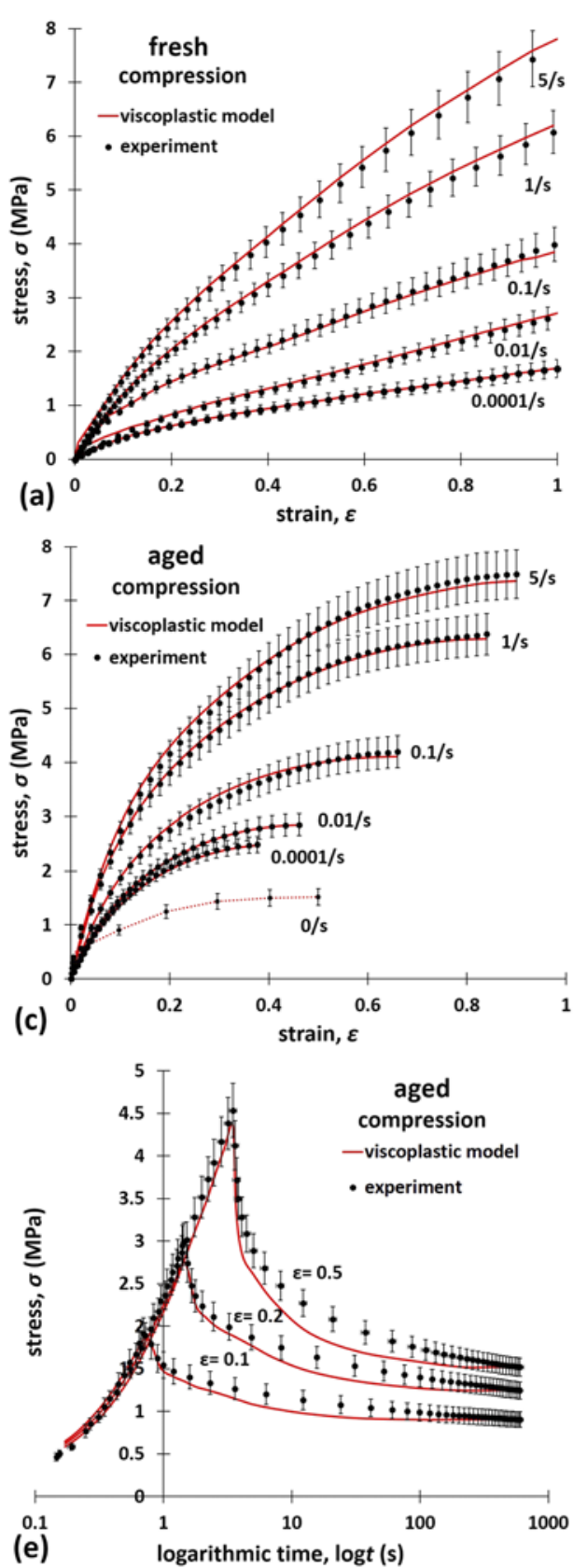
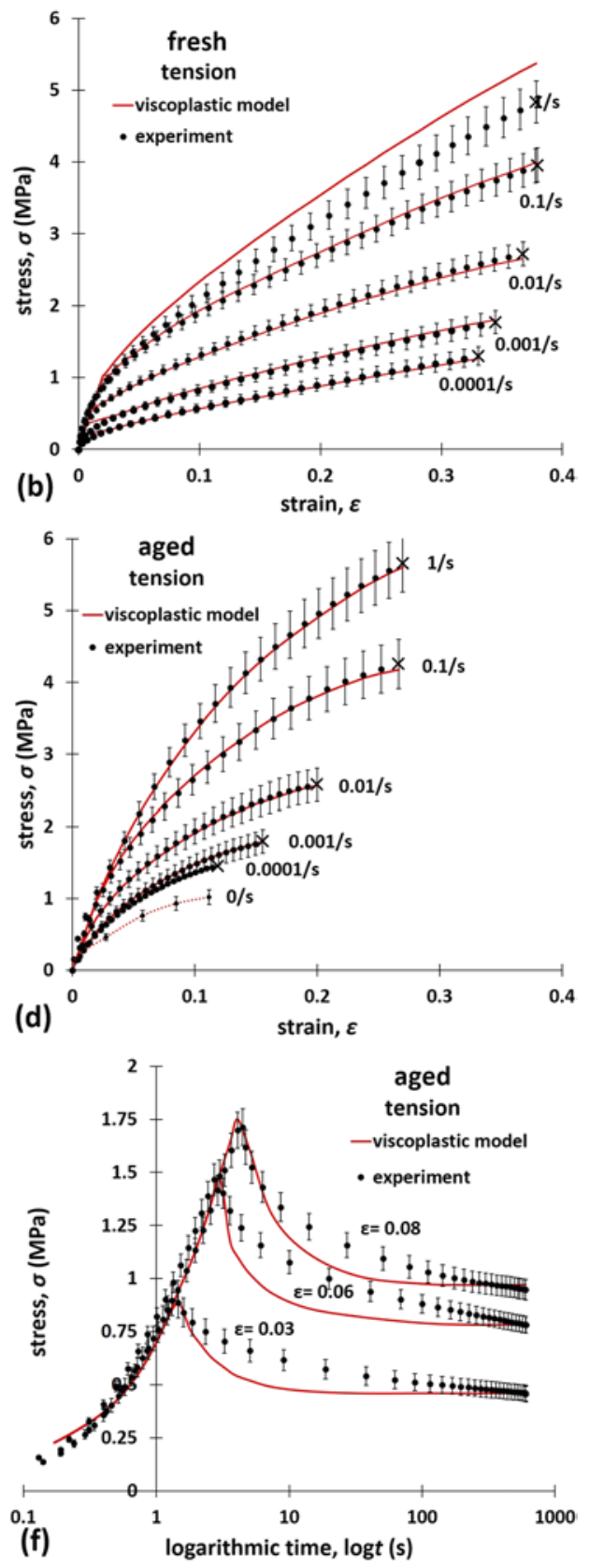

FIG. 2. Compression and tensile stressstrain data at various rates, between fresh and aged texture together with the viscoplastic model fit; (a) monotonic compression-fresh texture, (b) monotonic tension-fresh texture, (c) monotonic compression-aged texture, (d) monotonic tension-aged texture, (e) stress relaxation in compressionaged texture, (f) stress relaxation in tension-aged texture. The " $\mathrm{x}$ " marker of the last data point for each tensile curve denotes ultimate sample fracture/separation. The data points for the $0 \mathrm{~s}^{-1}$ rate in (c) and (d) correspond to the long term stresses obtained from relaxation tests at the corresponding constant strains. 


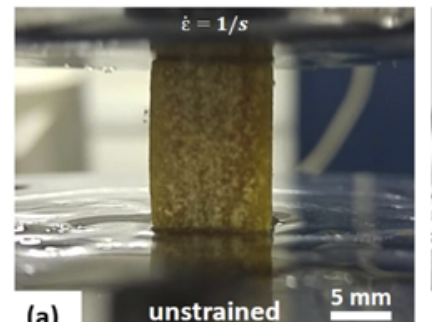

(a) unstrained

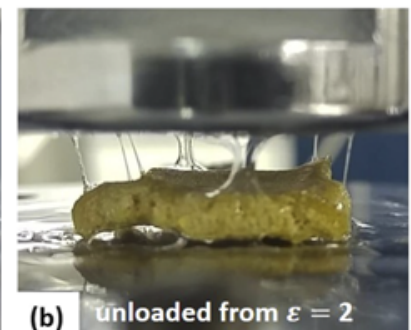

(b) unloaded from $\varepsilon=2$

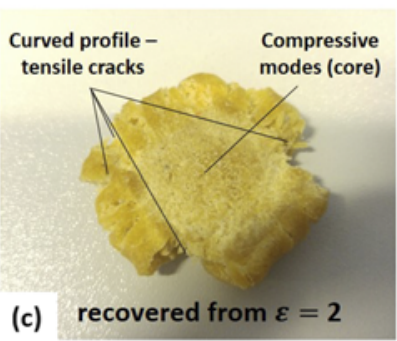

FIG. 3. Compressive behaviour of aged samples at $1 \mathrm{~s}^{-1}$ rate; (a) unstrained and (b) sample at the instance of unloading after the applied strain of 2 and (c) recovered sample ( $1 \mathrm{~h}$ after the test). other hand, freshness enhances ductility since significantly higher tensile strains at break, $\varepsilon_{f}$, are apparent [compare $\varepsilon_{f}$ values between Figs. 2(b) and 2(d)]. These $\varepsilon_{f}$ points increase markedly with rate in the aged texture, as opposed to the fresh material in which the effect is less pronounced, implying different failure mechanisms with age.

Importantly, the compressive response in the fresh texture did not show an ultimate failure point, i.e., the samples did not separate. Specifically, the forces kept increasing until the platens approached each other. However, due to the onset of sample barrelling approximately after the strain of 0.8 , the stress-strain data were considered valid only up to the strain level of 1, as plotted in Fig. 2(a) (Goh and Sherman, 1987). A similar compressive behaviour was found in the aged texture, where damage effects were also involved, as shown in Fig. 3 for the strain rate, $\dot{\varepsilon}=1 /$ s. Specifically, after a certain strain level, axial cracks are observed in the perimeter of the samples, leading to the final compressed profile shown in Fig. 3(c). Due to incompressibility $(v=0.5)$, the lateral tensile strains (sample lateral expansion) were significant $(50 \%$ of the axial strains) and comparable with the tensile failure, $\varepsilon_{f}$, values, promoting sample axial separation. On the other hand, evidence of sample barrelling in Fig. 3(c) suggests that in practise stresses may develop in the circumference of the sample, due to the expansion being constrained from friction. Indication of sample barrelling is the fact that the material close to the sample edges has a curved profile, while in the core region compressive modes of deformation can be seen. At lower $\dot{\varepsilon}$, the axial cracks initiated at significantly lower applied strains. This of course is due to the decreasing $\varepsilon_{f}$ with decreasing $\dot{\varepsilon}$ [see Fig. 2(d)]. Owing to this onset of cracking, a peak in the stress was calculated in all the rates, followed by decreasing values with further straining. Conclusively, in Fig. 2(c), the maximum stress-strain data points plotted correspond to these peak stresses. Beyond these points the curves were rendered invalid.

\section{B. Strain history dependence of failure strain}

As already mentioned in Sec. II B, additional relaxation tests were performed at strains of $0.11,0.13$, and 0.15 , as well as creep tests. The failure data obtained from these tests, as well as the failure points shown in Fig. 2(d), are used to define the dependence of $\varepsilon_{f}$ on the strain history. It was found that failure can even occur during a constant applied strain after some time elapses, i.e., during stress relaxation where $\dot{\varepsilon}==0 \mathrm{~s}^{-1}$. This behaviour is uniquely described in Fig. 4, via plotting all the $\varepsilon_{f}$ data obtained from the monotonic [Fig. 1(d)] relaxation and creep tests against a strain history factor, $\Omega$, defined as

$$
\Omega=\int_{0}^{t_{f}} \varepsilon d t,
$$

where $t_{f}$ is the breakage time measured from the point of strain application. Practically, $\Omega$ as a controlling parameter for $\varepsilon_{f}$ is a measure of both the duration and level of previously applied strain such that graphically it is equivalent to the area under the $\varepsilon-t$ curve until the point of breakage. Clearly, Fig. 4 displays a consistent logarithmic dependence on $\Omega$, regardless of the type of load application (monotonic or non-monotonic). Therefore, $\Omega$ renders itself a fundamental material parameter that can describe fracture in this soft solid and potentially to similar starch-based structures. While the concept of $\Omega$ is entirely new, its physical meaning may be linked to the micro-cracking mechanism in that upon applied strain the micro-cracks accumulate with time, promoting failure. Although long term fracture (low $\dot{\varepsilon}$, high $\Omega$ ) may not be relevant to the short time scales of oral breakdown (high $\dot{\varepsilon}$, low $\Omega$ ) reported in the literature (Gerstner et al., 2010; Skamniotis et al., 2016; and Wang and Chen, 2017), yet it may concern the markedly longer time scales reported for the gastric breakdown (Kong and Singh, 2008; Arora et al., 2005; and Bilecen et al., 2000). Moreover, while oral breakdown can be considered to occur through monotonic loading by the teeth, the same

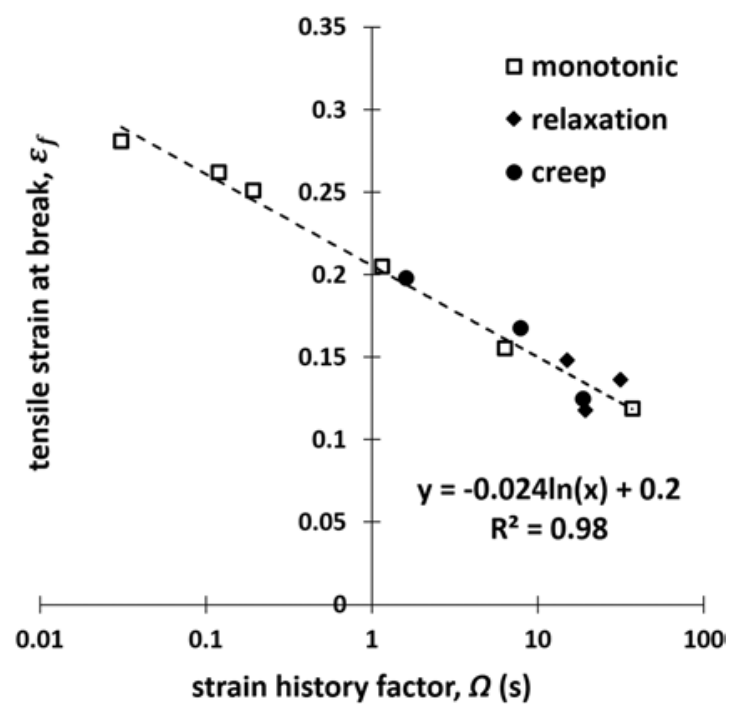

FIG. 4. Logarithmic dependency of tensile strain at break on the strain history factor, $\Omega$ [defined in Eq. (1)]. Monotonic, relaxation, and creep fracture data all fall on the same trendline. 
cannot be deemed for gastric breakdown, where the mechanical force history applied on the bolus has not been adequately quantified yet (Vassallo et al., 1992 and Cleary et al., 2015). Therefore, $\Omega$ is conveniently used here to define the damage onset criterion for the material model presented later in Sec. IV C.

\section{MATERIAL MODEL}

\section{A. Viscoplastic constitutive law and experimental calibration}

The viscoplastic material definition described in our previous work (Skamniotis et al., 2016) was also adopted here to capture the constitutive response of the aged starch food. Alternative material laws, such as the hyperviscoelastic laws (Simulia, 2013), were not found to provide an accurate simultaneous fit for both the compressive and tensile stress-strain curves. This is attributed to the significant damage through micro-cracking occurring in the pet food structure even at small tensile strains, not in agreement with the concept of hyperelasticity. Furthermore, the hyperviscoelastic laws are not compatible with any of the damage laws available in ABAQUS, which limited their applicability here in modeling fracture. Therefore the viscoplastic model is selected and is further enhanced with a ductile damage law (discussed in Sec. IV B) because it reproduces accurately the experimental stress-strain curves shown in Fig. 2.

The traditional viscoplastic law considers an initially elastic regime, followed by a Mises rate-dependent yield behaviour, which does not allow for a different stress-strain behaviour between compression and tension [e.g., Figs. 2(c) and 2(d)] to be modeled (Simulia, 2013). However, this limitation is surpassed here via extending the viscoplastic model through the ABAQUS/Explicit user defined field subroutine, VUSDFLD. The latter allows for additional dependencies to be introduced in the material yield data table (Simulia, 2013 and Rodríguez et al., 2013) extending the applicability of the model in a wider range of foods and materials in general. The dependency here is specified on the stress triaxiality factor, $\eta$, defined as

$$
\eta=\frac{\sigma_{H}}{\sigma_{e q}}
$$

where $\sigma_{H}$ is the hydrostatic stress and $\sigma_{e q}$ is the equivalent (von Mises) stress (Danas and Castañeda, 2012); for uniaxial compression $\eta=-1 / 3$, for simple shear $\eta=0$, while for uniaxial tension $\eta=1 / 3$. The $\eta$ parameter was calculated at the element integration points, based on the six stress tensor (3D case) components (Simulia, 2013). As a result, in the viscoplastic material definition, the $\sigma_{e q}-\varepsilon_{p_{e q}}$ test data (where $\varepsilon_{p_{e q}}$ is the equivalent plastic strain defined in the Appendix) for each equivalent plastic strain rate, $\dot{\varepsilon}_{p_{e q}}$, were tabulated for two $\eta$ values: $\eta=-1 / 3$ for compression and $\eta=1 / 3$ for tension. The dependency is concisely expressed as

$$
\sigma_{e q}=\sigma\left(\varepsilon_{p_{e q}}, \dot{\varepsilon}_{p_{e q}}, \eta\right) \text {. }
$$

The conversion from the $\sigma_{e q}-\varepsilon_{e q}$ data plotted in Fig. 2 into $\sigma_{e q}-\varepsilon_{p_{e q}}$ data was performed based on an initial modulus of $E=50 \mathrm{MPa}$ and the following equation:

$$
\varepsilon_{p_{e q}}=\varepsilon_{e q}-\frac{\sigma_{e q}}{E},
$$

while the plastic rates, $\dot{\varepsilon}_{p_{e q}}$, were set to be practically equal to the $\dot{\varepsilon}_{e q}$ rates, which are in turn equivalent to the tested rates, $\dot{\varepsilon}$, shown in Fig. 2. Based on differentiating Eq. (5), it can be deemed that the above condition is satisfied only when $E \gg \dot{\sigma}_{e q}$, which was the case here. Note that the $\dot{\varepsilon}_{p_{e q}} \approx \dot{\varepsilon}_{e q}$ condition is necessary in order to capture accurately the experimental stress-strain data in materials that exhibit a non-linear plastic regime. The monotonic and relaxation stress-strain model predictions are compared with the experimental data in Fig. 2. Figures 2(a)-2(d) demonstrate a very close agreement for the monotonic response both for the fresh and aged texture, respectively, while in Figs. 5(e) and 5(f) a reasonable fit is shown for stress relaxation for the aged texture.

However, as chewing generally imposes multiaxial stress states on the food item (Skamniotis et al., 2016 and Harrison et al., 2014b), an important concern was the response of the model for stress states other than uniaxial compression and tension. Specifically, outside the specified $\eta$ range between $-1 / 3$ and $1 / 3$, the model assumes a constant response, i.e., for biaxial compression $(\eta=-2 / 3)$, the $\sigma_{e q}-\varepsilon_{p_{e q}}$ behaviour is treated as equal to that corresponding to uniaxial compression. Although, it is not certain whether this assumption is indeed true, it was not possible to conduct multi-axial tests on the pet food samples. On the other hand, for stress states within
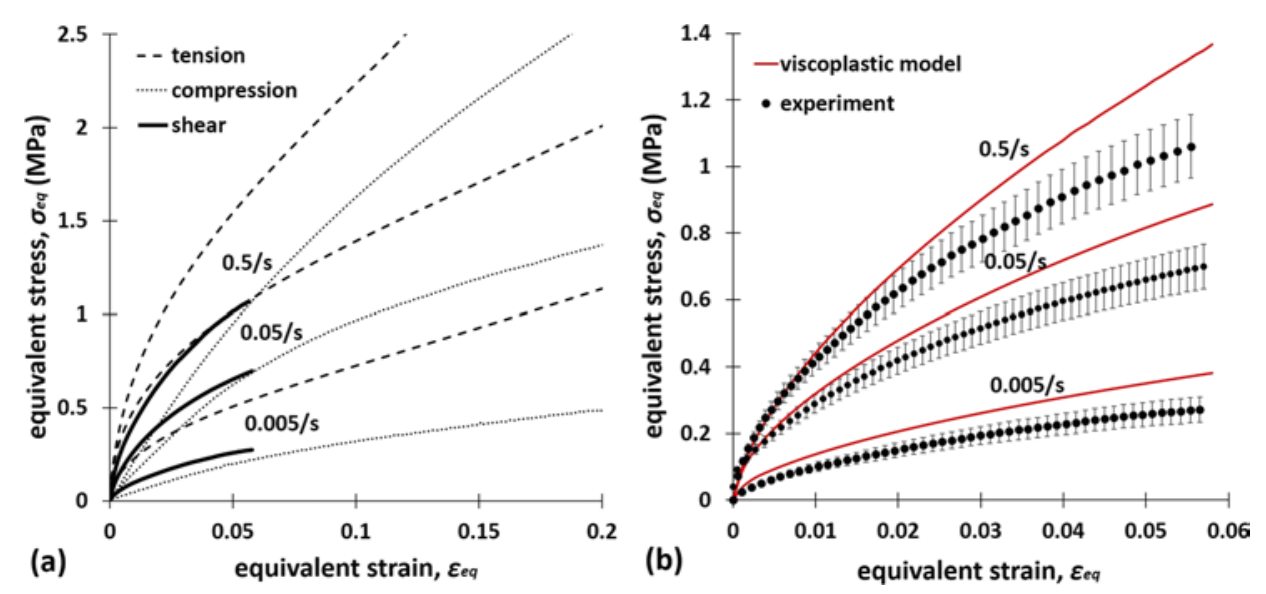

FIG. 5. Shear response; (a) comparison of $\sigma_{e q}-\varepsilon_{e q}$ data obtained via uniaxial tension, simple shear, and uniaxial compression tests on the fresh texture, for the three rates: $0.005 \mathrm{~s}^{-1}, 0.05 \mathrm{~s}^{-1}$, and $0.5 \mathrm{~s}^{-1}$, (b) comparison of the shear data against the viscoplastic model prediction for the three rates. 
the specified $\eta$ range, ABAQUS interpolates linearly, i.e., for shear $(\eta=0)$, the $\sigma_{e q}-\varepsilon_{p_{e q}}$ response is treated as the average between compression and tension.

The above assumption was verified through an independent simple shear experiment at $0.005 \mathrm{~s}^{-1}, 0.05 \mathrm{~s}^{-1}$, and $0.5 \mathrm{~s}^{-1}$ shear strain rates, using a shear sandwich configuration, as reported in Legghe et al. (2011) attached on a Dynamic Mechanical Analysis (DMA) testing machine (Buzza et al., 1995). Fresh rectangular samples of square cross sectional area, $A=8 \times 8 \mathrm{~mm}^{2}$, and thickness, $T=4 \mathrm{~mm}$, were sheared via serrated clamps, which allowed for valid data to be obtained up to a maximum shear strain, $\gamma$, of 0.1 ; beyond the latter value, slippage effects were significant. The shear stress, $\tau$, versus shear strain, $\gamma$, data were obtained through (Segal, 2002)

$$
\tau=\frac{F}{A}, \quad \gamma=\frac{\delta}{T},
$$

where $F$ and $\delta$ are the applied force and displacement, respectively. Comparison of these data with tension and compression required conversion of $\tau-\gamma$ into $\sigma_{e q}-\varepsilon_{e q}$ curves via the following expressions:

$$
\sigma_{e q}=\tau \sqrt{3}, \quad \varepsilon_{e q}=\gamma \frac{1}{\sqrt{3}} .
$$

This implies that the maximum applied $\gamma=0.1$ corresponds to a maximum $\varepsilon_{e q}=0.58$ for the shear data. The results are depicted in Fig. 5(a), which reveals that the $\sigma_{e q}-\varepsilon_{e q}$ curve obtained from shear lies between the ones derived from compression and tension, for all the three rates. In Fig. 5(b), the shear data are further compared with the viscoplastic model ABAQUS predictions obtained through single element simulations. The shear test data give lower stresses than the model, consistently for the three rates. This may be attributed to potential slippage effects even at low strains which however could not be quantified. For this reason, although the model curves can be adjusted to match the experimental data, this was not considered necessary. Nevertheless, the agreement shown in Fig. 5(b) is judged satisfactory for the purposes of the study, giving rise to the tested assumption and adding further credibility to the extended viscoplastic model.

\section{B. Ductile damage law framework}

A ductile damage law was chosen to model fracture, as it can be conveniently added to the viscoplastic law definition (Simulia, 2013). In addition, this damage law allows for modeling fracture initiation and propagation in continuum elements, based on an input fracture toughness value (Hillerborg et al., 1976) without the need to predefine the crack path.
Here, a value of $G_{c}=0.93 \mathrm{~kJ} / \mathrm{m}^{2}$ was used, obtained experimentally as the true material toughness for this material (aged texture), in recent work by the authors Skamniotis et al. (2017b). This is implemented through a stress-displacement, $\sigma_{e q}-u$, law, as the example shown in Fig. 6(a).

The example displays an initial elastic-plastic constitutive law with an elastic modulus, $E$, and a yield onset stress, $\sigma_{y 0}$, followed by a damage regime. Damage onset and full damage (element deletion) correspond to $D=0$ and $D=1$ values for the scalar damage variable, $D$, as shown in Fig. $6(\mathrm{~b})$. The displacement, $u$, is calculated via the characteristic element length, $L_{c h}$, as a measure of the element size, to give

$$
u=L_{c h} \varepsilon_{e q},
$$

where $\varepsilon_{e q}$ is the equivalent strain; the latter is associated with a equivalent plastic strain, $\varepsilon_{p_{e q}}$. Therefore, the shaded triangular area under the damage evolution response shown in Fig. 6(a) represents material toughness $G_{c}\left(\mathrm{~kJ} / \mathrm{m}^{2}\right)$, such that for a linear damage evolution law,

$$
u_{f}-u_{0}=\frac{2 G_{c}}{\sigma_{e q 0}},
$$

where $u_{f}, u_{0}$, and $\sigma_{e q 0}$ are displacement at full damage, displacement at damage onset, and equivalent stress at damage onset, respectively. Finally $D$ is updated through

$$
\dot{D}=\frac{\dot{u}}{u_{f}-u_{0}} \text {. }
$$

\section{Damage onset criterion}

In this section, the damage onset criterion is modified such that it can account for the different failure response expected under compression, shear, and tension. At the same time, the effect of the strain history (Sec. III B) is also captured. The new criterion assumes that damage is triggered by tensile strains. Therefore, the calibration of the failure criterion was performed using the tensile strain at break, $\varepsilon_{f}$ data (aged texture), shown in Fig. 2(d). This is based on previous work of the authors which found that when damage was allowed to occur under compression, unrealistic removal of material took place in tooth indentation simulations (Skamniotis et al., 2016). On the other hand, in contrast to uniaxial compression, shear does involve a positive maximum principal (tensile) stress, $\sigma_{1}$, which does raise potential towards material separation along $45^{\circ}$ to the shear plane (Dunne and Petrinic, 2005). Therefore, damage is here allowed to occur under shear as well as tensile states.
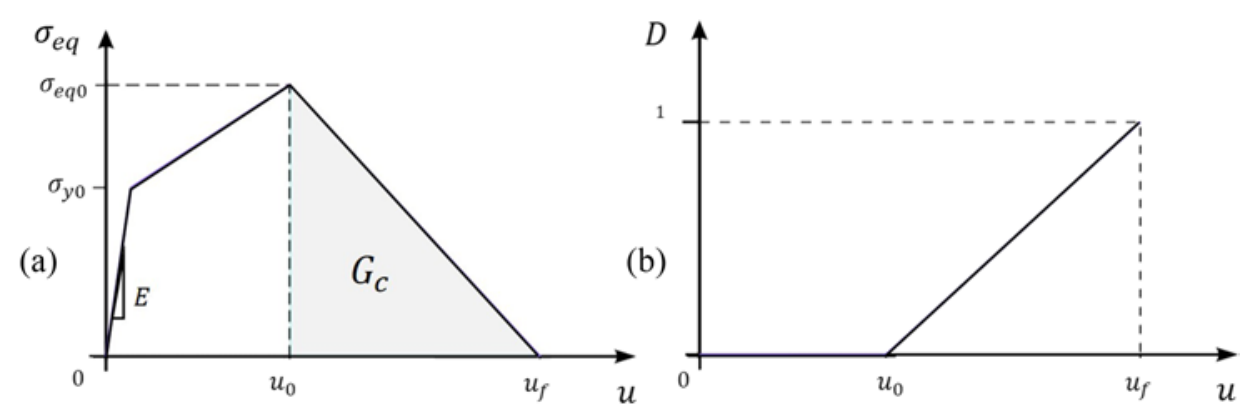

FIG. 6. Ductile damage law for an element in tension; (a) $\sigma_{e q}$ versus displacement, $u$, response with $G_{c}$ dissipation (shaded area under the graph), (b) corresponding damage variable, $D$, evolution versus $u$. 
The damage onset values, $\varepsilon_{p_{e q}}$, are specified as a function of $\eta$ and rate, $\dot{\varepsilon}_{p_{e q}}$; note again that here $\dot{\varepsilon}_{p_{e q}} \approx \dot{\varepsilon}_{e q}=\dot{\varepsilon}$. For $\eta=1 / 3$, the $\varepsilon_{p_{e q 0}}$ values were taken directly from Fig. 2(b) as a function of strain rate. For $\eta=0$, the $\varepsilon_{p_{e q 0}}$ values were specified such that damage onset occurs when the maximum principal strain, $\varepsilon_{1}$, reaches the tensile failure strain, $\varepsilon_{f}$, for each rate; this is explained further in the Appendix $\left(\varepsilon_{e q 0}\right.$ $=\frac{2}{\sqrt{3}} \varepsilon_{f}$ ). In order to prevent elements under compressive states from undergoing damage, an arbitrary value of $\varepsilon_{p e q 0}=2$ was used for $\eta \leq-1 / 3$, which is large enough such that it is never exceeded in the FE simulation (Skamniotis et al., 2016). A linear interpolation of $\varepsilon_{p_{e q 0}}$ between $\eta=0$ and $\eta=1 / 3$ and between $\eta=-1 / 3$ and $\eta=0$ is performed, while the $\varepsilon_{p_{e q 0}}$ values for each $\dot{\varepsilon}_{p_{e q}}$ are assumed constant for $\eta>1 / 3$. For clarity, the values of $\varepsilon_{p_{e q 0}}$, as a function of $\eta$ and rate, $\dot{\varepsilon}_{p_{e q}}$, are depicted in Fig. 7.

Finally, the dependence of failure strain, $\varepsilon_{f}$, on strain history characterised by $\varepsilon_{f}(\Omega)$ (see Fig. 4) was implemented in the new criterion. This is achieved via calculating the maximum principal strain, $\varepsilon_{1}$, and strain history factor, $\Omega$, in a subroutine (VUSDFLD) leading to the following expression:

$$
\text { IF } \eta \geq 0 \text { and } \varepsilon_{1} \geq \varepsilon_{f}(\Omega) \stackrel{\underset{\mathrm{ELSE}}{\longrightarrow} f_{1}=0}{\stackrel{\mathrm{THEN}}{\longrightarrow} f_{1}=1} \text {, }
$$

where $\varepsilon_{f}(\Omega)=-0.024 \ln \Omega+0.2$ (see Fig. 4) and $f_{1}$ is a field variable that determines whether damage occurs $\left(f_{1}=1\right)$ or not $\left(f_{1}=0\right)$. The latter is finally used as an additional independent variable in the tabular input for the criterion. Specifically, the arbitrary large value of $\varepsilon_{p_{e q 0}}=10$ was inputted for $f_{1}=0$, to ensure no damage in the FE model. In contrast, $\varepsilon_{p_{e q 0}}=0$ was entered for $f_{1}=1$, to guarantee damage. The new criterion described by Eq. (11) replaces the conventional criterion based on equivalent plastic strain, $\varepsilon_{p_{e q} 0}$, damage onset values, illustrated in Fig. 7.

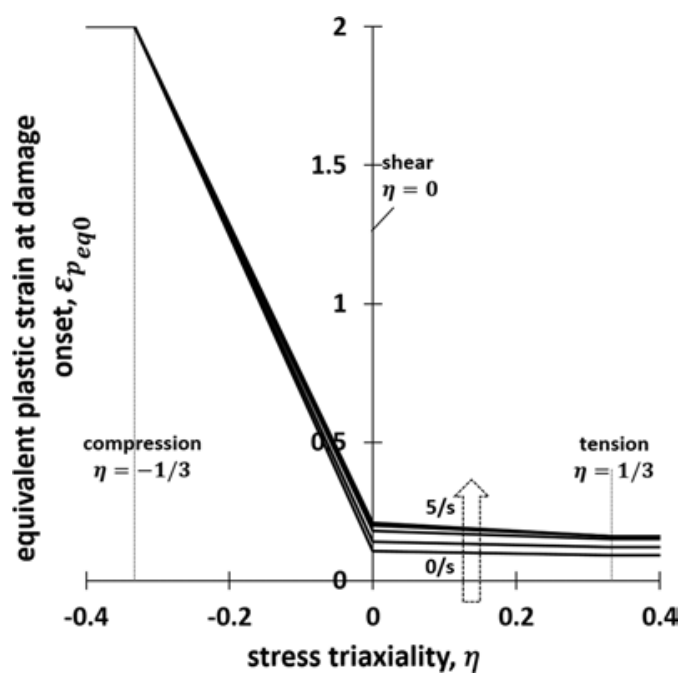

FIG. 7. Illustration of the initially used damage onset criterion for the ductile damage law.

\section{Damage evolution}

Under shear states, integration of the $\sigma_{e q}-L_{c h} \varepsilon_{e q}$ response [equal to $G_{c}$, see Fig. 6(a)], carries the contribution of both the minimum principal (compressive) stress, $\sigma_{3}<0$, and strain, $\varepsilon_{3}<0$, components. These compressive components, in fact, do not contribute to fracture in mode I (crack opening mode), for which the experimental $G_{c}$ value was obtained in Skamniotis et al. (2017b). Therefore, $G_{c}$ needs to also vary with stress triaxiality, $\eta$, which is achieved via the ratios,

$$
\left.\begin{array}{c}
\text { (a) } R_{\sigma}=\frac{\sigma_{e q 0}}{\sigma_{0}} \\
\text { (b) } R_{\varepsilon}=\frac{\varepsilon_{e q 0}}{\varepsilon_{f}}=\frac{\varepsilon_{e q f}}{\varepsilon_{F}}
\end{array}\right\},
$$

where $\sigma_{0}=\sigma_{1_{0}}$ and $\varepsilon_{f}=\varepsilon_{1}$ are the maximum principal stress and maximum principal strain (also tensile strain at break) at damage onset $(D=0)$, respectively, while $\varepsilon_{F}$ is the maximum principal strain at element deletion $(D=1)$ corresponding to an equivalent strain value, $\varepsilon_{\text {eqf }}$. Therefore, by considering that for a given $\eta, G_{\breve{c}}(\eta)$ is still calculated through Eqs. (8) and (9), the following relation can be deduced:

$$
\begin{aligned}
G_{\breve{c}}(\eta) & =\frac{1}{2} L_{c h} \sigma_{e q 0}\left(\varepsilon_{e q f}-\varepsilon_{e q 0}\right) \stackrel{(12)}{\Rightarrow} G_{\breve{c}}(\eta) \\
& =R_{\sigma} R_{\varepsilon} \frac{1}{2} L_{c h} \sigma_{0}\left(\varepsilon_{F}-\varepsilon_{f}\right) .
\end{aligned}
$$

Equation (13) is simplified by considering that for tension the following relations hold: $\sigma_{0}=\sigma_{e q 0}, \varepsilon_{f}=\varepsilon_{e q 0}$, and $\varepsilon_{F}=\varepsilon_{e q f}$, giving rise to the more fundamental equation

$$
G_{\breve{c}}(\eta)=R_{\sigma} R_{\varepsilon} G_{c},
$$

where $G_{c}$ is the experimental mode I fracture toughness. $G_{\breve{c}}(\eta)$ was implemented via Eqs. (12) and (14) in a subroutine (VUSDFLD). Equation (14) ensures that $G_{c}$ is dissipated along the maximum principal stress-maximum principal strain direction. Note that in tension $R_{\sigma}=R_{\varepsilon}=1$ and $G_{\breve{c}}(1 / 3)=G_{c}$ $=0.93 \mathrm{~kJ} / \mathrm{m}^{2}$, while in shear, $R_{\sigma}=\sqrt{3}$ and $R_{\varepsilon}=\frac{2}{\sqrt{3}}$, giving $G_{\breve{c}}(0) \approx 1.82 \mathrm{~kJ} / \mathrm{m}^{2}$. The latter can be deemed as the mode II (in-plane shear) toughness (Bárány et al., 2010), which is usually found to be larger than the mode I toughness (Benzeggagh and Kenane, 1996). Mode II toughness measurements for starch foods will be considered in future studies, in order to further validate the model, though such tests are a considerable experimental challenge (Nairn et al., 2017).

\section{APPLICATION OF MATERIAL MODEL TO FOOD BREAKDOWN STUDIES}

\section{A. Experimental and FE model setup}

The experimental apparatus included a Hitachi S-3400N SEM equipment, a micro-test rig with a $200 \mathrm{~N}$ load cell, rectangular samples cut at $15 \times 8 \times 4 \mathrm{~mm}$ (length $\times$ height $\times$ thickness) from aged square profile extrudates (Sec. II A), a rigid indenter of $R=4 \mathrm{~mm}$, and a rigid support platen (see Fig. 8). Two repeats were performed with an open SEM vacuum chamber, in order to obtain the force-displacement, $F-\delta$, data free of potential vacuum effects, i.e., loss of moisture; a variation of $4 \%$ in the area under the $F-\delta$ data was observed. Thereafter, one in situ repeat was performed, 


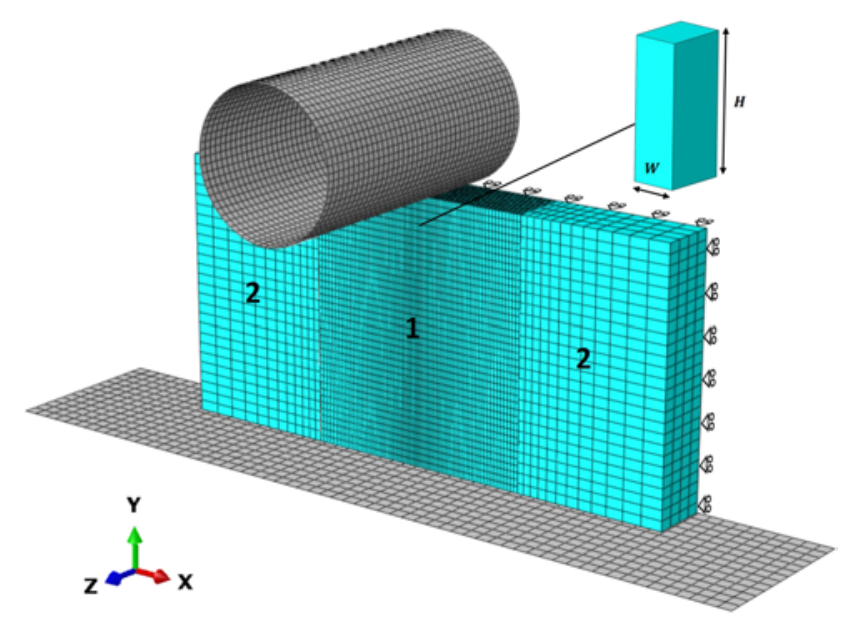

FIG. 8. FE model assembly of the verification test; an indication of the element aspect ratios is provided.

under vacuum conditions; the difference in the $F-\delta$ results between the vacuum and non-vacuum conditions was found insignificant.

The maximum allowed crosshead speed of $\dot{\delta}=0.042$ $\mathrm{mm} / \mathrm{s}$ was applied to the cylinder both in experiment and model, leading to a test duration (time until specimen separation) of approximately $\Delta t=100 \mathrm{~s}$. The FE model shown in Fig. 8 included a 3D mesh simulating the sample and discrete rigid shell parts for the support platen and the cylinder. Half of the specimen thickness was modeled through applying Z-symmetry boundary conditions (Goh et al., 2005), as denoted in Fig. 8. The cylinder was constrained in all translations and rotations except for the translation in the Y-axis, along which $\dot{\delta}=0.042 \mathrm{~mm} / \mathrm{s}$ was applied, whereas the platen remained fixed. The mesh consisted of 63335 3D stressdisplacement reduced integration hexahedral linear eight node (C3D8R) elements (Simulia, 2013), with a tie constraint between the coarse and fine mesh regions, denoted as " 2 " and " 1 " in Fig. 8, respectively. Poisson's ratio of $v=0.475$ was used instead of the true $v=0.5$, in order to avoid convergence problems (Simulia, 2013). Excessive element distortion due to large compressive strains was alleviated via selecting initial element aspect ratios, $H / W \approx 3$ (height, $H$, and over width, $W$ ) as displayed in Fig. 8 (Wanigasooriya, 2006 and Skamniotis et al., 2016). A mesh sensitivity analysis indicated that the model gives practically mesh independent $F-\delta$ results in the $W$ range $0.05-0.1 \mathrm{~mm}$. The ABAQUS/Explicit general contact algorithm was used, such that all the interior and exterior element facets are active in contact (Skamniotis et al., 2016). Due to unknown interaction properties, the effect of friction coefficient, $\mu$, on the $F-\delta$ data was studied for $\mu=0,0.2$, and 0.8 . Finally, $G_{c}=0 \mathrm{~kJ} / \mathrm{m}^{2}$ was specified for the elements of the sample's external surfaces, through an ABAQUS predefined field

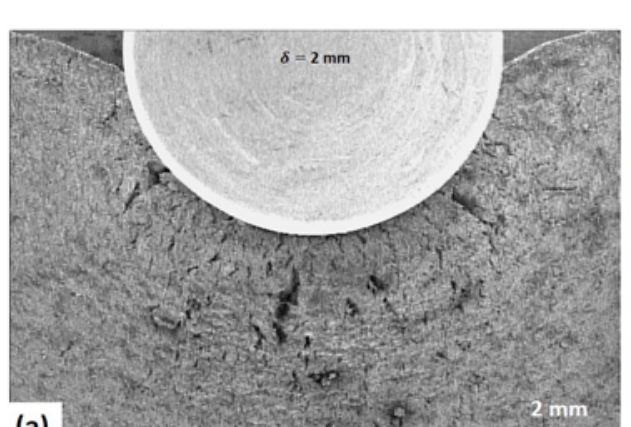

(a)
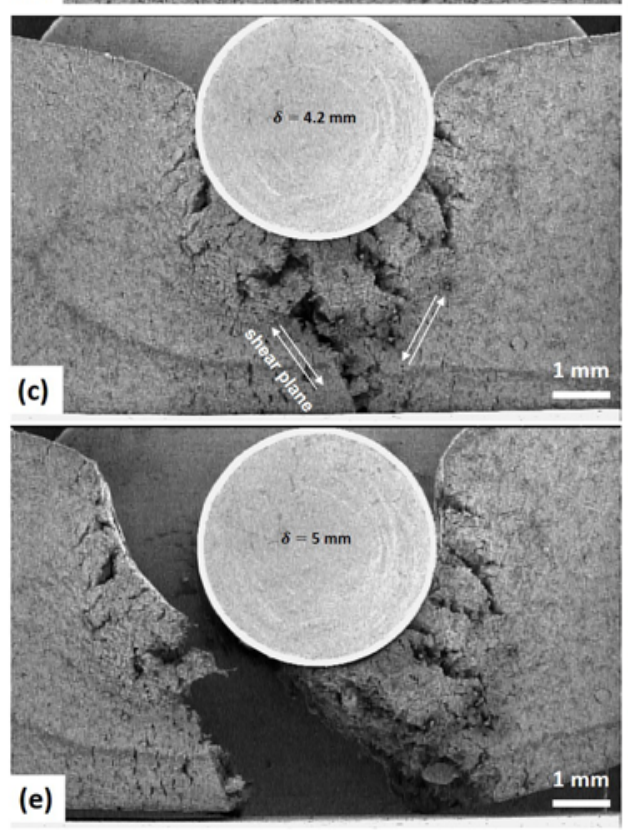

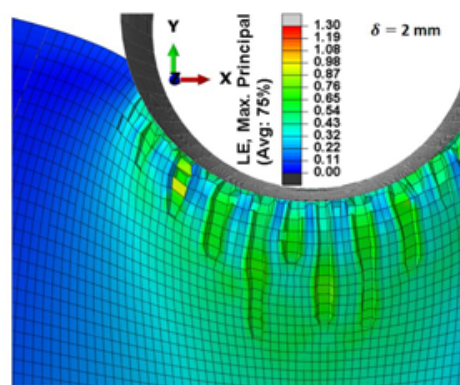

(b)
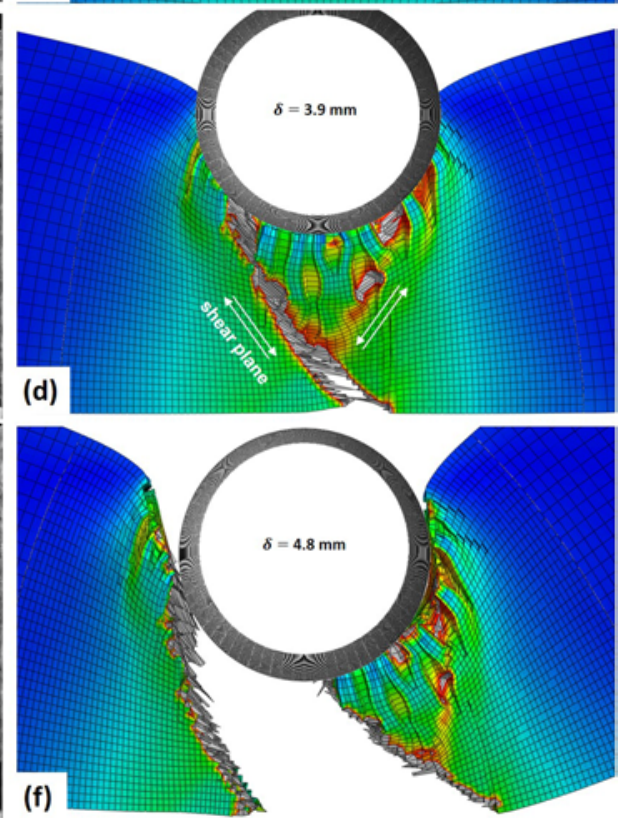

FIG. 9. In situ SEM frames of the indentation test in comparison with the FE results for progressive displacement, $\delta$ (aged texture-3 weeks old); the frames were chosen such that the overall damage state is comparable: (a) initial tensile cracks at $\delta=2 \mathrm{~mm}$, (b) FE: initial element deletions due to tensile strains at $\delta=2 \mathrm{~mm}$, (c) multiple cracks around the indenter and formation of a shear (inclined) plane at $\delta=$ $4.2 \mathrm{~mm}$, (d) FE: damage evolution in elements around the indenter and high tensile strains along two shear planes at $\delta=3.9 \mathrm{~mm}$, (e) final separation along one of the two shear planes at $\delta=5$ $\mathrm{mm}$, (f) FE: sample separation at $\delta=4.8$ $\mathrm{mm}$, similar to the experiment shown in (e); contours show maximum principal strain. 


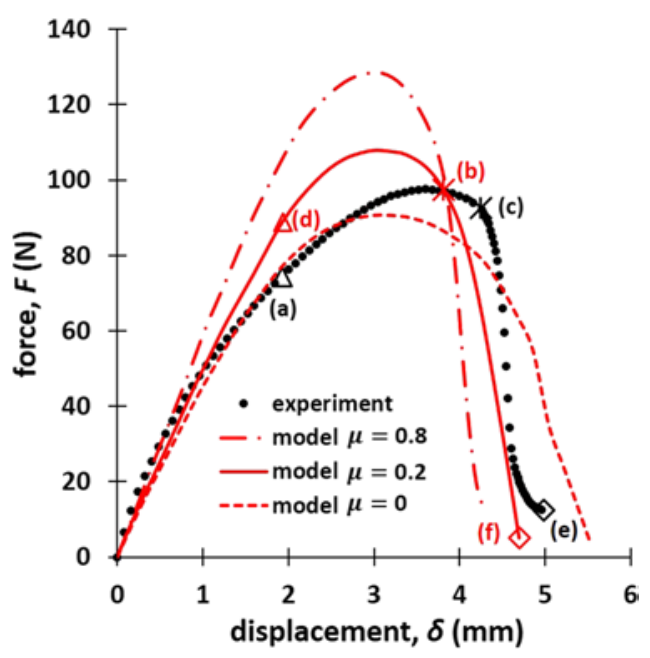

FIG. 10. Comparison of the experimental and FE force-displacement data; the FE results were obtained for friction coefficients, $\mu=0$ (frictionless), 0.2, and 0.8; the points (a)-(f) correspond to the frames shown in Fig. 11.

variable (Simulia, 2013). The latter was necessary in order to induce the required strain localisation such that accurate crack propagation results can be obtained via the ductile damage law.

\section{B. FE model results}

The FE results for $\mu=0.2$ and the in situ experimental indentation response are compared in Fig. 9. The respective $F-\delta$ data are depicted in Fig. 10. The merits of the material model are apparent, as a close agreement is shown not only in terms of the damage mechanism (Fig. 9) but also in terms of the $F-\delta$ response (Fig. 10). Specifically, the tensile cracks initiating circumferentially around the indenter [Fig. 9(a)] are modeled via "steep" element deletions as soon as the damage onset is met [Fig. 9(b)]. Note that tensile cracks are caused by the significant out-of-plane (along Z-axis) deformation of the sample due to material incompressibility. The bulk of the specimen is found to experience shear deformations while pure tension is more profound on the specimen surface, where the material is laterally unconstrained (plane stress). On the other hand, compression occurs in the core of the specimen (middle of thickness), particularly underneath the indenter were the material is confined (plane strain). The tensile cracks grow for increasing displacement in Fig. 9(c), which also reveals an inclined sample separation path, suggesting fracture along a shear deformation plane [denoted in Fig. 9(c)]. Finally, Fig. 9(e) shows sample separation along one of these shear planes, which is accurately reproduced by the FE model in Fig. 9(f). This fracture mechanism highlights the significance of modeling damage accurately for shear states, adding further credibility to the enhancements described in Secs. IV C and IV D.

The FE model also reveals the effect of friction on the overall response, as in Fig. 10 increasing $\mu$ gives a considerably stiffer $F-\delta$ response with lower failure displacement. For high friction $(\mu=0.8)$, the material was highly constrained to move with the indenter, which enhanced the shear strains, promoting damage, as evidenced by the smaller displacements for ultimate failure. Furthermore, barrelling occurred at sample regions close to the bottom platen. In contrast, for frictionless conditions $(\mu=0)$, the material around the indenter was free to expand laterally, while the shear strains were reduced, allowing for a larger displacement until sample separation (see Fig. 10). On the other hand, a coefficient of $\mu=0.2$ seems to be a more realistic value and this was used for comparison with the experiment. Summarising, friction is an important parameter for large strain indentation (Oldfield et al., 2013), having an effect on the stress states applied on the food.

Similar friction effects and stress states are also present in chewing (Skamniotis et al., 2016; Harrison et al., 2014a; and Harrison et al., 2014b), suggesting that this cylindrical indentation test, to some extent, resembles the mastication process. Therefore, it proves a useful method for food quality control and texture optimisation towards healthcare and palatable products. Furthermore, the model can aid in predictions of food particle size distribution in the oral cavity
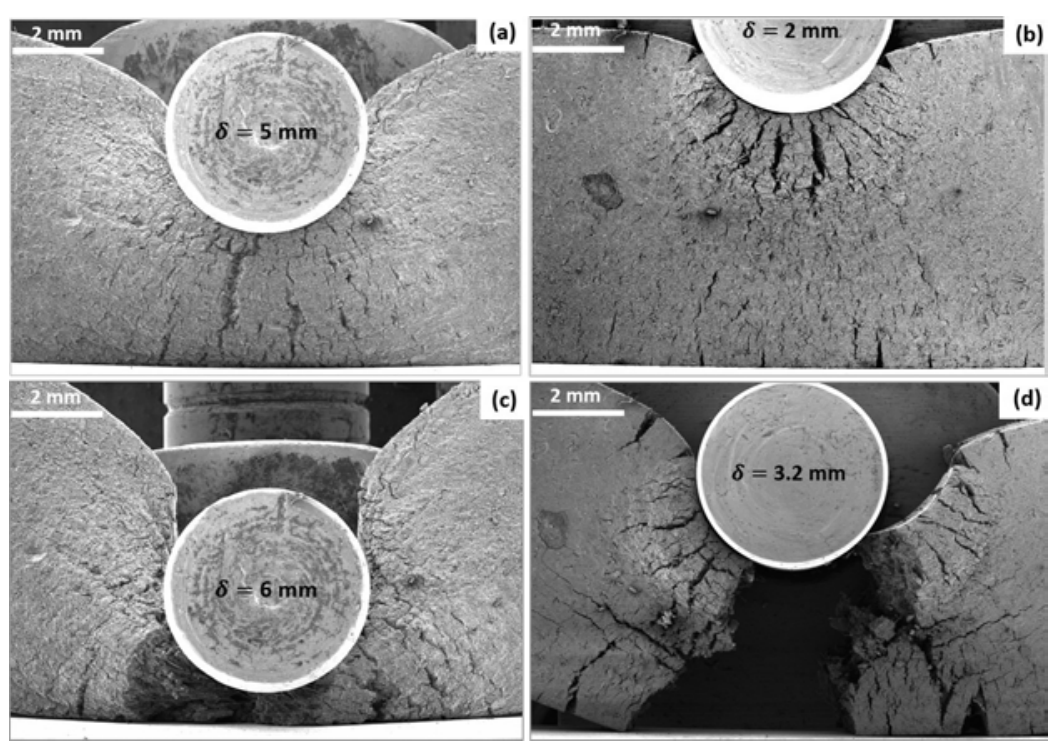

FIG. 11. Comparison of the cylindrical indentation response between the fresh (three days old) and the overaged (three months old) texture, through in situ SEM tests; (a) fresh sample at $\delta=5 \mathrm{~mm}$, (b) over-aged sample at $\delta=2 \mathrm{~mm}$, (c) fresh sample at maximum applied displacement, $\delta=7 \mathrm{~mm}$, (d) over-aged sample during ultimate separation at $\delta=3.5 \mathrm{~mm}$. 


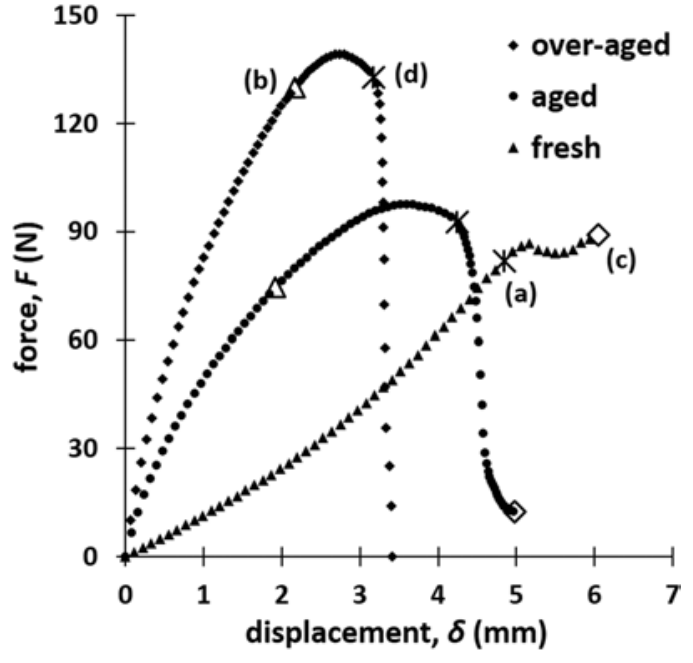

FIG. 12. Comparison of the force-displacement data between the three textures: over-aged, aged, and fresh; the points indicated correspond to the image numbers in Fig. 10.

(Le Révérend et al., 2016), as well as the associated number of chews needed to swallow (Le Révérend and Hartmann, 2014). Knowledge of the above mastication parameters is the first step towards developing multi-scale models of digestion (Cleary et al., 2015).

\section{Effect of food texture-age}

The effect of food texture as a result of ageing is demonstrated here experimentally via presenting two indentation responses of samples cut from a fresh (three days old) and an over-aged (three months old) texture of the square profile extrudates. These exhibited a soft-ductile and a hard-brittle response, as illustrated in Figs. 11(a) and 11(c) and Figs. 11(b) and $11(\mathrm{~d})$, respectively. The $F-\delta$ data (including the aged texture of Fig. 12) are summarised in Fig. 11. Clearly, the fresh pet food withstands large displacements, $\delta$, implying significant out-of-plane expansion [Fig. 11(a)], yet without complete sample separation [Fig. 11(c)]; the load increases further until the indenter touches the platen [see point (c) in Fig. 12]. This behaviour is attributed to the high failure strains of the fresh texture, shown in Fig. 2(b), as well as a toughness, $G_{c}$, likely even higher than $G_{c}=0.93 \mathrm{~kJ} / \mathrm{m}^{2}$ measured for the aged texture (Skamniotis et al., 2017b). The opposite phenomena are apparent in the over-aged samples, where multiple cracks initiate at small $\delta$ [Fig. 11(b)], followed by relatively brittle fracture [Fig. 11(d)], corresponding to a steep load drop in Fig. 12.

\section{CONCLUSIONS}

This study addresses the need for understanding and predicting the breakdown mechanism of complex soft solid structures such as starchy foods, under multi-axial loading conditions, towards gaining control over the process of digestion. The viscoplastic-damage material model developed here already delivers accurate results which will provide a vital basis to gain further knowledge on the above aspects and resolve a range of scientific and technological questions. The merits of the newly developed model over previous relevant FE models are as follows:

- Arbitrary crack paths can be predicted in a straightforward manner, i.e., without interrupting and restarting the FE analysis.

- The crack initiation and propagation behaviours thoroughly rely on the experimental tensile failure strain and fracture toughness values, respectively.

- Multi-axial loading conditions can be taken into account since a different stress-strain response and toughness parameter can be assigned to an element as a function of the applied stress state (stress triaxiality)/ deformation mode.

- A novel damage onset criterion is defined based on experimental results, i.e., a strain history dependent tensile failure strain.

- The requirement for minimal material removal (element deletion) is carefully addressed.

These enhancements were necessary here in obtaining accurate breakdown simulations in starch. The new viscoplastic-damage model also suggests that future product optimisation processes can be effectively performed through an FE model and fundamental mechanical tests, i.e., stressstrain and fracture toughness tests. Although the model was developed and calibrated for extruded starch, the methodology is also generalizable to other systems. This is because the new viscoplastic constitutive law coupled with progressive damage can reproduce a variety of stress-strain behaviours as a function of strain rate through a simple calibration process. When multi-axial loading conditions occur, the model can be extended through the stress triaxiality parameter and thus vary the stress-strain response depending on the stress state applied. The latter concerns materials which display significant differences between compression and tension, such as rubbers, polymers, and especially fibre reinforced systems. Therefore, the inclusion of stress triaxiality in the traditional viscoplastic law performed here, at the same time, inspires further in-house development of the existing builtin ABAQUS library as well as of other commercial FE software.

The novel material model can be further utilised in studying food disintegration and bolus breakdown and its relation with the original mechanical, physical, and chemical properties of products. Such studies aid in manufacturing targeted food product solutions for nutrition and metabolic health. The degradation of mechanical properties due to saliva hydration and acidic, enzymatic conditions of the gastric secretions, can be also included via adding dependencies on the material model parameters, i.e., stress-strain and toughness. Finally, the work presented here is the first step towards developing predictive models for the breakdown of soft solid foods during the oral or gastric process. Such models can be used to optimise consumer perception and/or nutrient bioavailability, respectively, by enabling the optimal material parameters to be determined in each case. Controlled food breakdown may be linked to flavour release and texture during the oral process whereas for the gastric process it will influence gastric emptying, satiety, and nutrient uptake. 


\section{ACKNOWLEDGMENTS}

The authors would like to acknowledge Mars Petcare for the financial support and for providing the samples. In addition, the SEM in situ micro-test device that was used in this work was purchased through funds from the Engineering and Physical Sciences Research Council (EP/C520629/1).

\section{APPENDIX: PRINCIPAL STRAINS FOR TENSION, SHEAR, AND COMPRESSION}

In Sec. III C, the calculation of $\varepsilon_{p_{e q 0}}$ such that damage onset occurs when $\varepsilon_{1}$ reaches $\varepsilon_{f}$, for each strain rate, is based on the following definition for $\boldsymbol{\varepsilon}_{\boldsymbol{e}}$ :

$\varepsilon_{e q}=\frac{\sqrt{2}}{3}\left[\sqrt{\left(\varepsilon_{1}-\varepsilon_{2}\right)^{2}+\left(\varepsilon_{2}-\varepsilon_{3}\right)^{2}+\left(\varepsilon_{3}-\varepsilon_{1}\right)^{2}}\right]$,

where $\varepsilon_{1}, \varepsilon_{2}$, and $\varepsilon_{3}$ are the maximum, intermediate, and minimum principal strains, respectively. These values become $\varepsilon_{1}=\varepsilon_{1_{0}}, \varepsilon_{2}=\varepsilon_{2_{0}}, \varepsilon_{3}=\varepsilon_{3_{0}}$, and $\varepsilon_{e q}=\varepsilon_{e q 0}$, at damage onset, such that in tension, Eq. (A1) gives $\varepsilon_{e q 0}=\varepsilon_{1_{0}}=\varepsilon_{f}$. In simple shear, $\varepsilon_{2}=0$ and thus $\varepsilon_{1_{0}}=-\varepsilon_{3_{0}}=\varepsilon_{f}$, such that Eq. (A1) gives $\varepsilon_{e q 0}=\frac{2}{\sqrt{3}} \varepsilon_{f}$. In compression, substituting $\varepsilon_{3_{0}}=-2 \varepsilon_{1_{0}}=-2 \varepsilon_{f}$ (incompressibility) and $\varepsilon_{1_{0}}=\varepsilon_{2_{0}}=\varepsilon_{f}$ into Eq. (A1) deduces $\varepsilon_{e q 0}=2 \varepsilon_{f}$. Thereafter, the above $\varepsilon_{e q 0}$ results for compression, shear, and tension were substituted into Eq. (5) to find the corresponding $\varepsilon_{p_{e q 0}}$ values at damage onset (shown in Fig. 7).

Agrawal, K. R. and Lucas, P. W., "The mechanics of the first bite," Proc. R. Soc. B 270(1521), 1277-1282 (2003).

Agrawal, K. R., Lucas, P. W., Prinz, J. F., and Bruce, I. C., "Mechanical properties of foods responsible for resisting food breakdown in the human mouth," Arch. Oral Biol. 42(1), 1-9 (1997).

Amuthakkannan, P., Manikandan, V., Winowlin Jappes, J. T., and Uthayakumar, M., "Effect of fibre length and fibre content on mechanical properties of short basalt fibre reinforced polymer matrix composites," Mater. Phys. Mech. 16, 107-1117 (2013).

Arora, S., Ali, J., Ahuja, A., Khar, R. K., and Baboota, S., "Floating drug delivery systems: A review," AAPS PharmSciTech 6(3), E372-E390 (2005).

Baragar, F. A., Van der Bilt, A., and Van Der Glas, H. W., "An analytic probability density for particle size in human mastication," J. Theor. Biol. 181(2), 169-178 (1996).

Bárány, T., Czigány, T., and Karger-Kocsis, J., "Application of the essential work of fracture (EWF) concept for polymers, related blends and composites: A review,” Prog. Polym. Sci. 35(10), 1257-1287 (2010).

Benzeggagh, M. L. and Kenane, M., "Measurement of mixed-mode delamination fracture toughness of unidirectional glass/epoxy composites with mixed-mode bending apparatus," Compos. Sci. Technol. 56(4), 439-449 (1996).

Berthaume, M. A., "Food mechanical properties and dietary ecology," Am. J. Phys. Anthropol. 159(S61), 79-104 (2016a).

Berthaume, M. A., "On the relationship between tooth shape and masticatory efficiency: A finite element study," Anat. Rec. 299(5), 679-687 (2016b).

Berthaume, M. A., Dumont, E. R., Godfrey, L. R., and Grosse, I. R., "How does tooth cusp radius of curvature affect brittle food item processing?," J. R. Soc., Interface 10(84), 20130240 (2013).

Bierer, T. L., "The role of biometrics in canine oral health and nutrition," Vet. Med. 1 (2007).

Bilecen, D., Scheffler, K., Seifritz, E., Bongartz, G., and Steinbrich, W., "Hydro-MRI for the visualization of gastric wall motility using RARE magnetic resonance imaging sequences," Abdom. Imaging 25(1), 30-34 (2000).

Bornhorst, G. M. and Singh, R. P., "Bolus formation and disintegration during digestion of food carbohydrates," Compr. Rev. Food Sci. Food Saf. 11(2), 101-118 (2012).
Buzza, D. M. A., Lu, C.-Y. D., and Cates, M. E., "Linear shear rheology of incompressible foams," J. Phys. II 5(1), 37-52 (1995).

Cardoso-Júnior, A., Coelho, L. G. V., Savassi-Rocha, P. R., Vignolo, M. C., Abrantes, M. M., De Almeida, A. M., Dias, E. E., Júnior, G. V., De Castro, M. M., and Lemos, Y. V., "Gastric emptying of solids and semisolids in morbidly obese and non-obese subjects: An assessment using the 13C-octanoic acid and 13C-acetic acid breath tests,' Obes. Surg. 17(2), 236-241 (2007).

Charalambides, M. N., Wanigasooriya, L., Williams, G. J., and Chakrabarti, S., "Biaxial deformation of dough using the bubble inflation technique. I. Experimental," Rheol. Acta 41(6), 532-540 (2002).

Charalambides, M. N., Wanigasooriya, L., Williams, J. G., Goh, S. M., and Chakrabarti, S., "Large deformation extensional rheology of bread dough," Rheol. Acta 46(2), 239-248 (2006).

Charalambides, M. N., Goh, S. M., Wanigasooriya, L., Williams, J. G., and Xiao, W., "Effect of friction on uniaxial compression of bread dough," J. Mater. Sci. 40(13), 3375-3381 (2005).

Cleary, P. W., Sinnott, M. D., Hari, B., Bakalis, S., and Harrison, S. M., "Modelling food digestion," in Modeling Food Processing Operations, Woodhead Publishing Series in Food Science, Technology and Nutrition (Woodhead Publishing, 2015), pp. 255-305.

Cohen, M. A., Ellis, S. M., Le Roux, C. W., Batterham, R. L., Park, A., Patterson, M., Frost, G. S., Mohammad, A. G., and Bloom, S. R., "Oxyntomodulin suppresses appetite and reduces food intake in humans," J. Clin. Endocrinol. Metab. 88(10), 4696-4701 (2003).

Danas, K. and Castañeda, P. P., "Influence of the lode parameter and the stress triaxiality on the failure of elasto-plastic porous materials," Int. J. Solids Struct. 49(11), 1325-1342 (2012).

Drechsler, K. C. and Ferrua, M. J., "Modelling the breakdown mechanics of solid foods during gastric digestion," Food Res. Int. 88, 181-190 (2016).

Dunne, F. and Petrinic, N., Introduction to Computational Plasticity (Oxford University Press on Demand, 2005).

Eng, C. M., Lieberman, D. E., Zink, K. D., and Peters, M. A., "Bite force and occlusal stress production in hominin evolution," Am. J. Phys. Anthropol. 151(4), 544-557 (2013).

Epstein, B., "The mathematical description of certain breakage mechanisms leading to the logarithmico-normal distribution," J. Franklin Inst. 244(6), 471-477 (1947)

Forte, A. E., Galvan, S., Manieri, F., Rodriguez y Baena, F., and Dini, D., “A composite hydrogel for brain tissue phantoms," Mater. Des. 112, 227-238 (2016).

Gamonpilas, C., Charalambides, M. N., and Williams, J. G., "Determination of large deformation and fracture behaviour of starch gels from conventional and wire cutting experiments," J. Mater. Sci. 44(18), 4976-4986 (2009).

Gamonpilas, C., Charalambides, M. N., Williams, J. G., Dooling, P. J., and Gibbon, S. R., "Predicting the mechanical behaviour of starch gels through inverse analysis of indentation data," Appl. Rheol. 20(3), 33283 (2010).

German, A. J., Holden, S. L., Bissot, T., Morris, P. J., and Biourge, V., "A high protein high fibre diet improves weight loss in obese dogs," Vet. J. 183(3), 294-297 (2010).

Gerstner, G. E., Cooper, M., and Helvie, P., "Chewing rates among domestic dog breeds," J. Exp. Biol. 213(13), 2266-2272 (2010).

Goh, H. C. and Sherman, P., "Influence of surface friction on the stress relaxation of gouda cheese," J. Texture Stud. 18(4), 389-404 (1987).

Goh, S. M., "An engineering approach to food texture studies," Ph.D. thesis, Imperial College London, 2002.

Goh, S. M., Charalambides, M. N., and Williams, J. G., "On the mechanics of wire cutting of cheese," Eng. Fract. Mech. 72(6), 931-946 (2005).

Harrison, S. M., Cleary, P. W., Eyres, G., Sinnott, M. D., and Lundin, L., "Challenges in computational modelling of food breakdown and flavour release," Food Func. 5(11), 2792-2805 (2014a).

Harrison, S. M., Eyres, G., Cleary, P. W., Sinnott, M. D., Delahunty, C., and Lundin, L., "Computational modeling of food oral breakdown using smoothed particle hydrodynamics," J. Texture Stud. 45(2), 97-109 (2014b).

Hiiemae, K., Heath, M. R., Heath, G., Kazazoglu, E., Murray, J., Sapper, D., and Hamblett, K., "Natural bites, food consistency and feeding behaviour in man," Arch. Oral Biol. 41(2), 175-189 (1996).

Hillerborg, A., Modéer, M., and Petersson, P.-E., "Analysis of crack formation and crack growth in concrete by means of fracture mechanics and finite elements," Cem. Concr. Res. 6(6), 773-781 (1976). 
Kong, F. and Singh, R. P., "Disintegration of solid foods in human stomach," J. Food Sci. 73(5), R67 (2008).

Kozu, H., Kobayashi, I., Nakajima, M., Uemura, K., Sato, S., and Ichikawa, S., "Analysis of flow phenomena in gastric contents induced by human gastric peristalsis using CFD," Food Biophys. 5(4), 330-336 (2010).

Kupczik, K. and Stynder, D. D., "Tooth root morphology as an indicator for dietary specialization in carnivores (Mammalia: Carnivora)," Biol. J. Linn. Soc. 105(2), 456-471 (2012).

Le Révérend, B. and Hartmann, C., "Numerical modeling of human mastication, a simplistic view to design foods adapted to mastication abilities," Physiol. Behav. 124, 61-64 (2014).

Le Révérend, B., Saucy, F., Moser, M., and Loret, C., "Adaptation of mastication mechanics and eating behaviour to small differences in food texture," Physiol. Behav. 165, 136-145 (2016).

Legghe, E., Joliff, Y., Belec, L., and Aragon, E., "Computational analysis of a three-layer pipeline coating: Internal stresses generated during the manufacturing process," Comput. Mater. Sci. 50(4), 1533-1542 (2011).

Lentle, R. G., Janssen, P. W. M., DeLoubens, C., Lim, Y. F., Hulls, C., and Chambers, P., "Mucosal microfolds augment mixing at the wall of the distal ileum of the brushtail possum," Neurogastroenterol. Motil. 25(11), 881 (2013).

Liu, M., Meakin, P., and Huang, H., "Dissipative particle dynamics simulation of multiphase fluid flow in microchannels and microchannel networks," Phys. Fluids 19(3), 033302 (2007).

Loret, C., Walter, M., Pineau, N., Peyron, M. A., Hartmann, C., and Martin, N., "Physical and related sensory properties of a swallowable bolus," Physiol. Behav. 104(5), 855-864 (2011).

Lucas, P. W. and Luke, D. A., "Computer simulation of the breakdown of carrot particles during human mastication," Arch. Oral Biol. 28(9), 821-826 (1983a).

Lucas, P. W. and Luke, D. A., "Methods for analysing the breakdown of food in human mastication," Arch. Oral Biol. 28(9), 813-819 (1983b).

Luke, D. A. and Lucas, P. W., "The significance of cusps," J. Oral Rehabil. 10(3), 197-206 (1983).

McCarthy, C. T., Ní Annaidh, A., and Gilchrist, M. D., "On the sharpness of straight edge blades in cutting soft solids: Part II-Analysis of blade geometry," Eng. Fract. Mech. 77(3), 437-451 (2010).

Mohagheghian, I., Wang, Y., Zhou, J., Yu, L., Guo, X., Yan, Y., Charalambides, M. N., and Dear, J. P., "Deformation and damage mechanisms of laminated glass windows subjected to high velocity soft impact," Int. J. Solids Struct. 109, 46 (2017).

Mohammed, M. A. P., Tarleton, E., Charalambides, M. N., and Williams, J. G., "Mechanical characterization and micromechanical modeling of bread dough," J. Rheol. 57(1), 249-272 (2013).

Mohammed, M. A. P., Tarleton, E., Charalambides, M. N., and Williams, J. G., "Mechanical characterization and micromechanical modeling of bread dough," J. Rheol. 57(1), 249-272 (2013).

Moscicki, L., Mitrus, M., Wojtowicz, A., Oniszczuk, T., and Rejak, A., Extrusion-Cooking of Starch (Advances in Agrophysical Research, 2013).

Moxon, T. E., Gouseti, O., and Bakalis, S., "In silico modelling of mass transfer \& absorption in the human gut," J. Food Eng. 176, 110-120 (2016).

Nairn, J. A., Hammerquist, C., and Aimene, Y. E., "Numerical implementation of anisotropic damage mechanics," Int. J. Numer. Methods Eng. (published online, 2017).
Norton, I., Moore, S., and Fryer, P., "Understanding food structuring and breakdown: engineering approaches to obesity," Obes. Rev. 8 (s1), 83-88 (2007).

Oldfield, M. J., Dini, D., Jaiswal, T., and Rodriguez y Baena, F., “The significance of rate dependency in blade insertions into a gelatin soft tissue phantom," Tribol. Int. 63, 226-234 (2013).

Pera, P., Bucca, C., Borro, P., Bernocco, C., De Lillo, A., and Carossa, S., "Influence of mastication on gastric emptying," J. Dental Res. 81(3), 179-181 (2002).

Ramanamurthy, J. V., Prasad, K. M., and Narla, V. K., "Unsteady peristaltic transport in curved channels," Phys. Fluids 25(9), 091903 (2013).

Rayner, C. K., Samsom, M., Jones, K. L., and Horowitz, M., "Relationships of upper gastrointestinal motor and sensory function with glycemic control," Diabetes Care 24(2), 371-381 (2001).

Rodríguez Soler, J., Martinez Cutillas, F. J., and Rodriguez, J. M., "Concrete constitutive model, calibration and applications," presented at the 2013 SIMULIA Community Conference, 2013.

Segal, V. M., "Severe plastic deformation: Simple shear versus pure shear," Mater. Sci. Eng.: A 338(1), 331-344 (2002).

Simulia, Abaqus Version, 6.13 Documentation, Dassault Systemes, 2013.

Skamniotis, C. G., Patel, Y., Charalambides, M. N., and Elliott, M., "Fracture investigation in starch-based foods," Interface Focus 6(3), 20160005 (2016).

Skamniotis, C. G., Charalambides, M. N., and Elliott, M., "Chewing as a forming application: A viscoplastic damage law in modelling food oral breakdown," AIP Conf. Proc. 1896, 150002 (2017a).

Skamniotis, C. G., Kamaludin, M. A., Elliott, M., and Charalambides, M. N., "A novel essential work of fracture experimental methodology for highly dissipative materials," Polymer 117, 167 (2017b).

Taghipoor, M., Lescoat, P., Licois, J.-R., Georgelin, C., and Barles, G., "Mathematical modeling of transport and degradation of feedstuffs in the small intestine," J. Theor. Biol. 294, 114-121 (2012).

Vandenberghe, E., Charalambides, M. N., Mohammed, I. K., De Ketelaere, B., De Baerdemaeker, J., and Claes, J., "Determination of a critical stress and distance criterion for crack propagation in cutting models of cheese," J. Food Eng. 208, 1-10 (2017).

Vassallo, M. J., Camilleri, M., Prather, C. M., Hanson, R. B., and Thomforde, G. M., "Measurement of axial forces during emptying from the human stomach," Am. J. Physiol.: Gastrointest. Liver Physiol. 263(2), G230-G239 (1992).

Vincent, J. F. V., "Application of fracture mechanics to the texture of food," Eng. Failure Anal. 11(5), 695-704 (2004).

Voon, F. C. T., Lucas, P. W., Chew, K. L., and Kuke, D. A., "A simulation approach to understanding the masticatory process," J. Theor. Biol. 119(3), 251-262 (1986).

Wang, X. and Chen, J., "Food oral processing: Recent developments and challenges," Curr. Opin. Colloid Interface Sci. 28, 22 (2017).

Wang, Y., Brasseur, J. G., Banco, G. G., Webb, A. G., Ailiani, A. C., and Neuberger, T., "A multiscale lattice Boltzmann model of macro-to microscale transport, with applications to gut function," Philos. Trans. R. Soc., A 368(1921), 2863-2880 (2010).

Wanigasooriya, L., Mechanical Characterisation and Ram Extrusion of Wheat Flour Dough (Imperial College London, 2006). 